\title{
The methyl-CpG-binding domain (MBD) is crucial for MeCP2's dysfunction-induced defects in adult newborn neurons
}

\author{
Na Zhao ${ }^{1,2 † \neq}$, Dongliang Ma ${ }^{1 \neq}$, Wan Ying Leong ${ }^{1}$, Ju Han ${ }^{1}$, Antonius VanDongen ${ }^{1,3}$, \\ Teng Chen ${ }^{2}$ and Eyleen L. K. Goh ${ }^{1,3,4 *}$

\begin{abstract}
Programme in Neuroscience and Behavioral Disorder, Duke-NUS Graduate Medical School, Singapore, Singapore, ${ }^{2}$ Key Laboratory of Health Ministry for Forensic Science, Department of Forensic Medicine, Xi'an Jiaotong University School of Medicine, Xi'an, Shaanxi, China, ${ }^{3}$ Department of Physiology, Yong Loo Lin School of Medicine, National University of
\end{abstract} \\ Singapore, Singapore, Singapore, ${ }^{4}$ KK Research Center, KK Women's and Children's Hospital, Singapore, Singapore
}

OPEN ACCESS

Edited by:

Laurie Doering,

McMaster University, Canada

Reviewed by:

Alexander K. Murashov, East Carolina University, USA Paola Tognini,

University of California Irvine, USA

${ }^{*}$ Correspondence:

Eyleen L. K. Goh,

Programme in Neuroscience and Behavioral Disorders, Duke-NUS Graduate Medical School, 8 College Road, Singapore 169857, Singapore Tel: 65-6516-6701, Fax: 65-6557-0729

eyleen.goh@duke-nus.edu.sg

${ }^{\dagger}$ Present address: Na Zhao, Northwest University of Politics and Law School of Police, Xi'an, China

${ }^{\ddagger}$ These authors have contributed equally to this work.

Received: 16 February 2015 Accepted: 08 April 2015

Published: 24 April 2015

Citation:

Zhao N, Ma D, Leong WY, Han J, VanDongen $A$, Chen $T$ and Goh ELK

(2015) The methyl-CpG-binding domain (MBD) is crucial for MeCP2's dysfunction-induced defects in adult newborn neurons.

Front. Cell. Neurosci. 9:158. doi: 10.3389/fncel.2015.00158
Mutations in the human X-linked gene MECP2 are responsible for most Rett syndrome $(\mathrm{RTT})$ cases, predominantly within its methyl-CpG-binding domain (MBD). To examine the role of $\mathrm{MBD}$ in the pathogenesis of $\mathrm{RTT}$, we generated two MeCP2 mutant constructs, one with a deletion of MBD (MeCP2- $\triangle \mathrm{MBD}$ ), another mimicking a mutation of threonine 158 within the MBD (MeCP2-T158M) found in RTT patients. MeCP2 knockdown resulted in a decrease in total dendrite length, branching, synapse number, as well as altered spontaneous $\mathrm{Ca}^{2+}$ oscillations in vitro, which could be reversed by expression of full length human MeCP2 (hMeCP2-FL). However, the expression of hMeCP2- $\triangle \mathrm{MBD}$ in MeCP2-silenced neurons did not rescue the changes in neuronal morphology and spontaneous $\mathrm{Ca}^{2+}$ oscillations, while expression of hMeCP2-T158M in these neurons could only rescue the decrease in dendrite length and branch number. In vivo over expression of $\mathrm{hMeCP} 2-\mathrm{FL}$ but not hMeCP2- $\triangle \mathrm{MBD}$ in adult newborn neurons of the dentate gyrus also rescued the cell autonomous effect caused by MeCP2 deficiency in dendrites length and branching. Our results demonstrate that an intact and functional MBD is crucial for MeCP2 functions in cultured hippocampal neurons and adult newborn neurons.

Keywords: Rett syndrome, newborn neurons, dendrites, methyl-CpG-binding domain, spontaneous $\mathrm{Ca}^{2+}$ oscillations

\section{Introduction}

Rett syndrome (RTT) is a X-linked neurological disorder affecting mostly females. Classical RTT is a progressive neurodevelopment disorder. Girls with RTT exhibit normal development through the first 6-18 month after birth, followed by an abrupt neuroregression and growth stagnation (Neul et al., 2010). Mutations within MECP2 (methyl-CpG binding protein 2) on the X-chromosome are responsible for nearly $95 \%$ of all RTT cases (Amir et al., 1999). MeCP2 is a member of the family of methyl-CpG binding domain (MBD) containing proteins that is the most abundant in post-mitotic neurons and it functions as a transcriptional regulator in the brain. MeCP2 contains a $\mathrm{N}$-terminal domain (NTD), a methyl-binding domain (MBD), an intervening domain (ID), a transcriptionalrepressor domain (TRD) and a C-terminal domain (CTD; Hansen et al., 2011). Common mutations 
found in RTT patients are primarily clustered within the MBD and the TRD of MeCP2 (Bienvenu and Chelly, 2006; Heckman et al., 2014). Patients with mutations in the MBD exhibit more severe clinical features than the mutations beyond this area (Fabio et al., 2014). The most common mutation found in RTT patients occurs at residue T158 located at the Cterminus of the MBD (Ballestar et al., 2005; Ghosh et al., 2010). Considering the key role of MBD in transcriptional function of MeCP2 and the high frequency of T158 mutations observed in RTT patients, the function of the MBD as well as the T158 mutation has become an important focus of many studies.

Abnormal levels of MeCP2 in the brains of mouse RTT disease models lead to RTT-like phenotypes including tremors, breathing abnormalities, hypoactivities and limb stereotypies. Like the human conditions, mice RTT models show an apparent normal early development before the onset of overt symptoms. After the onset of symptoms, the animals typically die at 10-12 weeks of age (Chen et al., 2001; Belichenko et al., 2008; Ricceri et al., 2013). Previous studies demonstrated that expression levels of $\mathrm{MeCP} 2$ in humans and rodents increase during neuronal development and maturation, suggesting MeCP2 may be important during the normal neuronal development and maturation (Shahbazian and Zoghbi, 2002; Nguyen et al., 2012; Ma et al., 2015). Studies in Xenopus also revealed a specific function of $\mathrm{MeCP} 2$ during early neural development (Stancheva et al., 2003; Marshak et al., 2012).

Brain autopsy material from RTT patients and MeCP2 mutant mice revealed normal gross anatomy without detectable loss of neurons but impaired dendritic growth and reduced complexity of pyramidal cells in the associate brain regions (Armstrong, 2005; Chapleau et al., 2009). This observation prompted the hypothesis that an underlying cause of RTT is a defect in neuronal and synaptic function. MeCP2 deficiency in cells and mice as well as cells from RTT patients are associated with changes in cellular and synaptic physiology (Marchetto et al., 2010; Ricciardi et al., 2011; Ma et al., 2015). However, it is still not clear if these cellular and synaptic changes and defects are cell-autonomous effects and if they are caused by the loss-of-function mutations in RTT neurons. Although it is possible to generate RTT mouse models with each individual human mutation identified, it will not be possible to distinguish between cell-autonomous and non-cell-autonomous (or secondary) effects of $\mathrm{MeCP} 2$ in neurons of these mice where $\mathrm{MeCP} 2$ is mutated or deleted in all cell types. Therefore, an in vivo system that allows genetic manipulation of individual cells in the brain is necessary to circumvent the limitations associated with all currently available MeCP2 knockout/knock-in mouse models of RTT.

Here we provide functional evidence on the MBDdependent role of MeCP2 in neuronal development in cultured hippocampal neurons. Full length $\mathrm{MeCP} 2$ and mutant MeCP2 containing either the MBD deletion or $\mathrm{T} 158 \rightarrow \mathrm{M}$ mutation were used to study morphological and functional roles of the MBD in these neurons. Short hairpin RNA (shRNA) was targeted to individual newborn granule neurons in adult brain to determine cell-autonomous effects of MeCP2 in vivo.

\section{Materials and Methods}

\section{Plasmid and Viral Production}

For MeCP2 knockdown, a short hairpin RNA (GGGAAAC TTGTTGTCAAGATGCC) was cloned under the control of the human U6 promoter with Tomato co-expressing under the Synapsin promoter. A shRNA with scrambled sequence was used as a control as described previously (Ma et al., 2015). In order to overexpress MeCP2 proteins in post-mitotic cells, a plasmid encoding the full length human wild-type MECP2 (hMeCP2-FL) was generated under the control of the Ubiquitin promoter ( $\mathrm{Ub})$ in the lentiviral FUGW vector. Constructs expressing an MBD deletion $(\triangle \mathrm{MBD})$ or $\mathrm{T} 158$ mutation $(\mathrm{T} 158 \mathrm{M})$ were generated using the same lentiviral backbone. To examine the functional role of $\mathrm{MeCP} 2$ and its mutants in structural plasticity in vivo, engineered selfinactivating murine retroviruses were used to express GFP specifically in proliferating cells and their progeny in the dentate gyrus of adult mice. hMeCP2-FL or $\triangle \mathrm{MBD}$ together with GFP were cloned under the control of EF1 $\alpha$ promoter with shMeCP2 co-expressed under the control of human U6 promoter in the same vector. Western analysis was performed to validate the specificity and efficiency of the different constructs.

A high titer of virus $\left(1 \times 10^{9}\right.$ unit $\left./ \mathrm{ml}\right)$ was produced by transfection of different constructs into HEK293gp cells using the calcium phosphate method as described previously (Ma et al., 2015). Briefly, constructs were mixed with $\mathrm{CaCl}_{2}$ and added to $2 \times$ HEPES buffer saline $(\mathrm{pH}=7.0)$. The DNA mix was incubated for $30 \mathrm{~min}$ and then added to the HEK293gp cells followed by ultracentrifugation of viral supernatant.

\section{Primary Hippocampal Neuronal Culture and Transfection}

Primary hippocampal neurons were isolated from embryonic day 18 (E18) Long-Evans rats and embryonic hippocampi were collected in buffer $(127 \mathrm{mM} \mathrm{NaCl}, 5 \mathrm{mM} \mathrm{KCl}, 170$ $\mu \mathrm{M} \mathrm{Na} \mathrm{HPO}_{4}, 205 \mu \mathrm{M} \mathrm{KH_{2 }} \mathrm{PO}_{4}, 5 \mathrm{mM}$ Glucose, $59 \mathrm{mM}$ Sucrose, $100 \mathrm{U} / \mathrm{mL}$ Penicillin/Streptomycin, $\mathrm{pH}$ 7.4). At least 3-4 batches of culture were used for each experiment, with at least 2 coverslips per batch. Each batch of cultures was isolated from pooled hippocampi of all E18 pups (typically 8-10) from 1 animal. Cells were dissociated with $25 \mathrm{mg} / \mathrm{ml}$ papain and plated on poly-L-lysine $(1 \mathrm{mg} / \mathrm{ml})$ coated coverslips or plates. High densities of cells (84000/well) were prepared for calcium imaging and low densities of cells (42000/well) were used for immunochemistry staining. Hippocampal neurons were cultured in Neurobasal medium (Invitrogen) supplemented with B-27, penicillin-streptomycin, L-glutamine at $37^{\circ} \mathrm{C}$. Neuronal cultures were infected with the lentiviral vector carrying the control shRNA (shctrl) or MeCP2 shRNA (shMeCP2) construct at DIV (day in vitro) 1. Lentiviral vector carrying the rescue construct (hMeCP2$\mathrm{FL} / \triangle \mathrm{MBD} / \mathrm{T} 158 \mathrm{M})$ was added immediately after the redfluorescent of shRNA was visualized $(48 \mathrm{~h}$ after shMeCP2 transfection). 


\section{Immunochemistry}

After DIV 12, hippocampal primary neurons were fixed for $30 \mathrm{~min}$ with $4 \%$ paraformaldehyde in $0.1 \mathrm{M}$ phosphate buffer (PB), washed with DPBS (Invitrogen) three times and then blocked with $5 \%$ normal donkey serum in $0.1 \%$ TBS-Triton (TBS-TX) buffer for $2 \mathrm{~h}$ at room temperature. Primary antibodies were diluted in blocking solution at $4^{\circ} \mathrm{C}$ using the following dilutions: 1:1000 rabbit anti-MAP2 (Millipore), 1:1000 mouse anti-MAP2 (Sigma), 1:1000 mouse anti-Synapsin-1 (Abcam), 1:500 rabbit anti-MeCP2 (Cell Signaling Technology) and 1:500 mouse anti-MeCP2 (Sigma). After incubation in primary antibodies overnight, coverslips were incubated in the appropriate secondary antibodies diluted in blocking solution for $2 \mathrm{~h}$ at room temperature.

The brain tissues were fixed 2 weeks after stereotaxic injection by vascular perfusion through the left ventricle of the heart with sequential delivery of $50 \mathrm{ml}$ of saline and $60 \mathrm{ml}$ of $4 \%$ paraformaldehyde in $0.1 \mathrm{M} \mathrm{PB}$. Coronal brain sections $(40 \mu \mathrm{m})$ were prepared and processed for immunostaining using the antiDCX (Santa Cruz; 1:300) antibodies.

\section{Imaging and Neuronal Morphology Analysis}

Both coverslips and brain slices were imaged on a Zeiss LSM 710 confocal system (Carl Zeiss) using a multi-track configuration. For in vitro studies, all values were obtained from at least 3 batches of culture of at least 2 coverslips per batch. At least 20 neurons from each coverslips were used for analysis. Quantification of the total dendritic length and total dendritic branch number as well as the number of synapsin-1 positive puncta of each cell were performed as previously described ( $\mathrm{Ng}$ et al., 2013; Ma et al., 2015). Images were semi-automatically traced with NIH ImageJ using the NeuronJ plugin, generating data for the total dendritic length and total dendritic branch number. Quantification of synapsin-1 puncta was done manually on a pre-determined length of traced dendrite, and then presented on graphs as the number of puncta per $\mu \mathrm{m}$ dendrite. For in vivo studies, three-dimensional reconstructions of the dendritic processes on brain slices were made from Z-series stacks of confocal images. The projection images were traced with ImageJ using the NeuronJ plugin and total dendritic length and total dendritic number of each virus infected new born neuron (DCX+) in the granule cell layer were analyzed. Acquisition of all images as well as morphology quantification was performed under "blind" conditions. The distributions of the total dendritic length and branch number of each individual neuron under different conditions were shown in accumulative distribution plots or bar plots. Cumulative frequency is used to determine the number of observations (e.g., total neurite length of each neuron) that lie above (or below) a particular value in a data set. The cumulative frequency is calculated using a frequency distribution table. This method is defined as the percentage of observations falling in each class interval. Relative cumulative frequency (\%) can be calculated by dividing the frequency of each interval by the total number of observations. An average of total 25-30 neurons from 4-6 animals (per experimental group) injected with virus carrying shRNA and/or expression construct were analyzed.

\section{Calcium Imaging and Peak Detection}

After DIV 12, hippocampal primary neurons were washed twice with loading buffer (118 mM NaCl, $4.69 \mathrm{mM} \mathrm{KCl}, 4.2 \mathrm{mM}$ $\mathrm{NaHCO}_{3}, 1.18 \mathrm{mM} \mathrm{KH} \mathrm{PO}_{4}, 0.8 \mathrm{mM} \mathrm{MgCl}$, $2.0 \mathrm{mM} \mathrm{CaCl}$, $20 \mathrm{mM}$ HEPES, $30 \mathrm{mM}$ glucose, $\mathrm{pH}=7.4$ ) and incubated with X-Rhod-1 (Molecular Probes/Invitrogen, Carlsbad, CA) with a final concentration of $1 \mu \mathrm{M}$ for $30 \mathrm{~min}$ at $37^{\circ} \mathrm{C}$. In order to remove excess dye, cells were washed with loading buffer twice and incubated for additional 20 min to equilibrate intracellular dye concentration and allow de-esterification of the dye. Timelapse image sequences of 500 frames were acquired with a region of $512 \times 512$ pixels, with $488 \mathrm{~nm}$ (FITC) and $534 \mathrm{~nm}$ filters on a LSM 710 inverted fluorescence confocal microscopy (Carl Zeiss, Pte. Ltd., Singapore). Images were acquired with ZEN software (Carl Zeiss Pte. Ltd., Singapore). At least 20 GFP positive neurons were randomly selected for calcium imaging (as indicated in figure legends) from at least 2 coverslips/batch.

All the data analysis was done using MATLAB (Mathworks, Natick, MA). For each coverslip, more than 20 cells were selected to record the calcium intensity under fluorescence with a sampling rate of $1.56 \mathrm{~s} /$ frame. Peak detection was done in MATLAB according to previous studies (Marchetto et al., 2010; Ma et al., 2015). The amplitude of each peak is measured by the difference between the peak value and the baseline.

\section{Animals and Stereotaxic Injection}

Ethics statement: All animal procedures and applicable regulations of animal welfare were in accordance with IACUC guidelines and approved by SingHealth IACUC, Singapore.

Adult (5-6 weeks old) female C57BL/6 mice were purchased from SingHealth Experimental Medicine Center (SEMC), Singapore, and kept in a temperature controlled environment $\left(22 \pm 2^{\circ} \mathrm{C}\right)$ with a 12 -h light/dark cycle. Animals were deeply anesthetized and stereotaxically injected at four sites $(0.5 \mu \mathrm{l}$ per site at $0.25 \mu \mathrm{l} / \mathrm{min}$ ) with the following coordinates (from bregma in $\mathrm{mm}$ ): anterioposterior, -2 ; lateral, \pm 1.5 ; ventral, 2.2; and anterioposterior, -3 ; lateral, \pm 2.5 ; ventral, 3 . A total of 60 animals were used and all efforts were made to reduce the number of animal used and also to minimize animal suffering.

\section{Statistical Analyses}

All the data were expressed as mean \pm SEM. Student's $t$ test for two groups' comparison and one-way ANOVA with a post hoc multiple comparison (Tukey test) were used to analyze the statistical significance between groups. The statistical significance was set at $P=0.05$.

\section{Results}

\section{Full-Length MeCP2 is Essential for the Maintenance of Normal Dendritic Development in Primary Hippocampal Neurons}

To investigate the role of endogenous MeCP2 in dendritic growth in cultured hippocampal neurons, we knocked down 
the expression of MeCP2 with lentivirus carrying shRNA against MeCP2 (Figure 1A). Endogenous MeCP2 protein level was significantly reduced in these neurons (Figures 1B,C). To confirm the efficiency of shMeCP2 in vivo, we generated retroviral construct carrying shMeCP2 (Figure 1A). An overexpression construct using the same retroviral backbone was also generated to express human MeCP2 (hMeCP2) that is not recognized by shMeCP2 specifically targeting mouse MeCP2 (Figure 1A). Retrovirus carrying either a knockdown construct or expression construct together with a fluorescence marker were stereotaxically injected into the border of hilus and DG where the progenitor cells are residing, as previously described (Ma et al., 2015) (Figures 1D-F). Retrovirus only infects dividing cells and therefore enables genetic manipulations (to express or knock down MeCP2) in individual progenitor cells in the adult hippocampus (Figures 1D-F). To confirm the neuronal identify of virus-infected cells, an immature neuronal marker, DCX was used for immunostaining (Figure 1E). The brain sections were also immunostained with antibody against $\mathrm{MeCP} 2$ to verify that virus-infected cells expressing fluorescence marker (GFP) express low or no MeCP2 (shMeCP2 expression construct) or express high MeCP2 (hMeCP2 expression construct) as compared to cells carrying shctrl expression construct (Figure 1F). This in vivo system also allows investigations on cell-autonomous effects of MeCP2 on neuronal development, which is not possible using any available knockout or knock-in RTT mouse models.

We next determined if an intact MBD in MeCP2 is essential for the maintenance of normal dendritic development in
A

Constructs for knockdown or overexpression of MeCP2 in vitro: pLenti-shctrl or shMeCP2 - U6, shMeCP2-Synapsin1-tdTomato-

pLenti-hMeCP2-FL pLenti-hMeCP2- $\triangle \mathrm{MBD}$ pLenti-hMeCP2-T158M

Construct for knockdown or overexpression of MeCP2 in vivo: pRetro-shctrl or shMeCP2 - U6 shMeCP2- EF1 - EGFP pRetro-hMeCP2-FL - U6 : shctrl - EF1 $\alpha$ EGFP hMeCP2-FL-

B

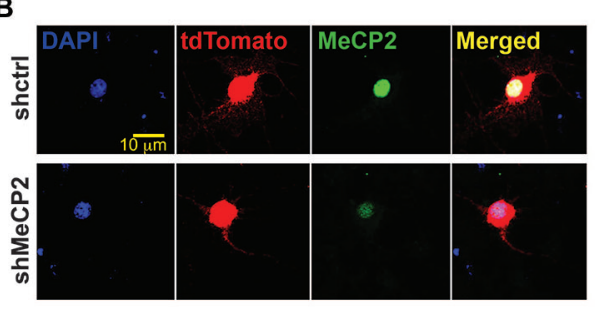

C

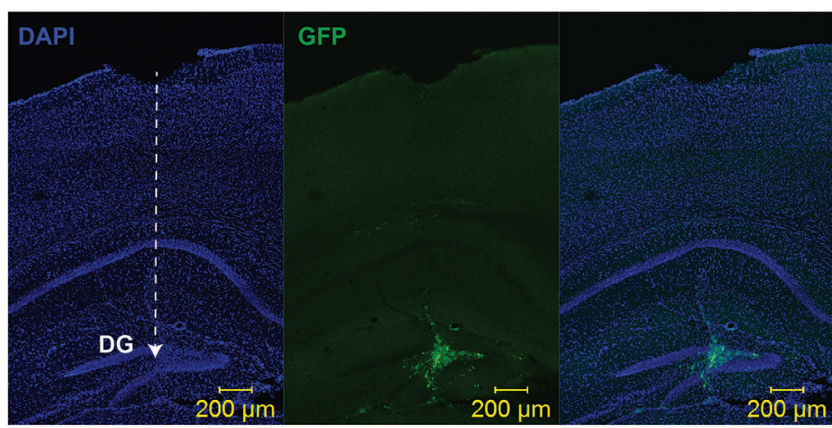

E

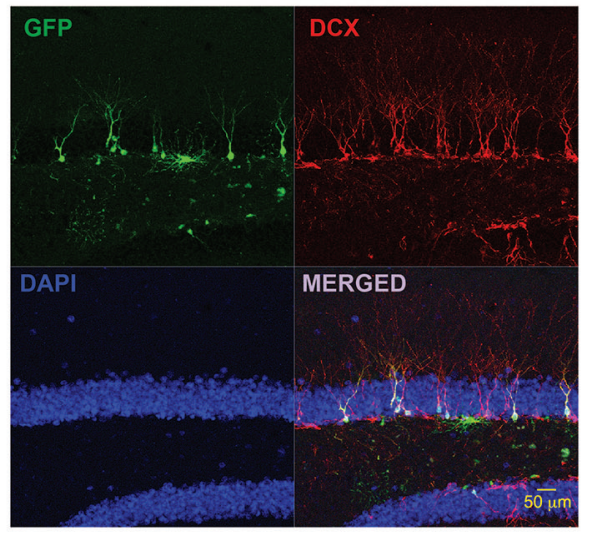

F
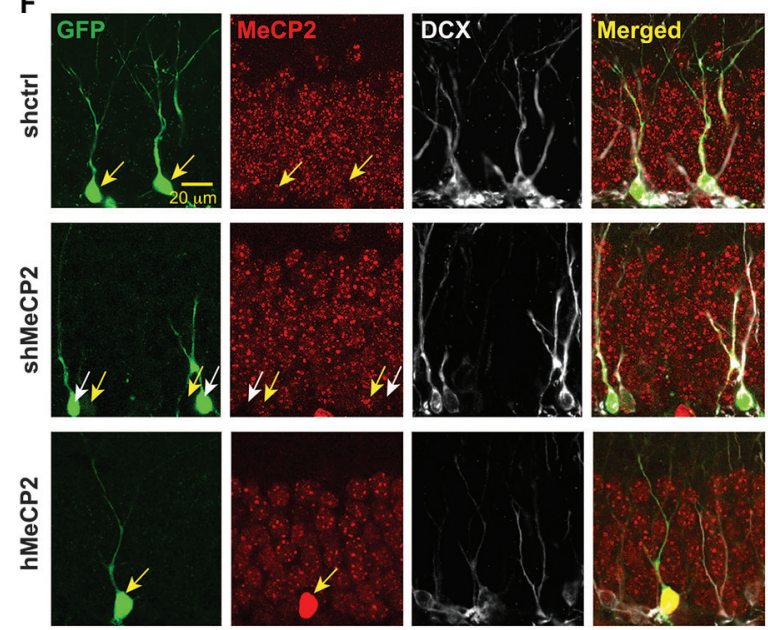

FIGURE 1 | Efficiency of MeCP2 knockdown in cultured primary hippocampal neurons. (A) Schematic diagram showing lentiviral constructs for knocking down MeCP2 or expressing hMeCP2 (FL, $\triangle \mathrm{MBD}$ or T158M) in vitro as well as retroviral constructs for checking the efficiency of MeCP2 knockdown or overexpression in progenitor cells in vivo. (B) Representative images from immunocytochemistry of $\mathrm{MeCP} 2$ in cultured hippocampal neurons at DIV 12 showing efficient knockdown of MeCP2 in vitro. (C) Quantification of fluorescence intensity of cells expressing shctrl or shMeCP2 that were immunostained with antibody against MeCP2 ${ }^{* * \star} p<0.001$, Student's $t$-test, $n=3$ batches). (D) Low magnification (10x) images showing the virus injection site and needle track (dashed white line with arrow pointed at the end point of injection) in dentate gyrus (DG) of the mouse brain. (E) High magnification (20x) images showing the virus infected neurons co-localized with DCX (immature neuron marker). (F) Representative images showing efficiency of MeCP2 knockdown or overexpression in progenitor cells in the DG in vivo (Immature neurons identified with anti-doublecortin (DCX)). Virus-infected neurons (green) and their MeCP2 expression (red) were indicated on images with corresponding yellow arrows. shMeCP2-expressing cells in middle panel (pointed by white arrows) show no or very low expression of MeCP2 demonstrating efficient knockdown. 
cultured hippocampal neurons. Co-expression of hMeCP2-FL but not hMeCP2- $\triangle \mathrm{MBD}$ or hMeCP2-T158M with shctrl, moderately decreased the total dendritic length and branch number in control neurons (Figures 2A,B), indicating a loss of function of both hMeCP2- $\triangle \mathrm{MBD}$ and hMeCP2-T158M. As expected, the total dendritic length and total branch number were significantly decreased in MeCP2-silenced neurons $\left({ }^{* * *} p<0.001\right.$ ) (Figures 2C,D), indicating that MeCP2 is necessary for dendrite development of these neurons. However, only the expression of hMeCP2-FL and hMeCP2-T158M in $\mathrm{MeCP} 2$ silenced neurons were effective in rescuing the MeCP2-deficiency-associated dendritic outgrowth defects (Figures 2C,D). In contrast, hMeCP2- $\triangle \mathrm{MBD}$ overexpression had no effects on MeCP2 silenced neurons (Figures 2C,D). This indicates hMeCP2-T158M may still have residual functions as compared to hMeCP2- $\triangle \mathrm{MBD}$.

\section{Intact MBD Domain in MeCP2 is Essential for Synapse Formation}

We next determined if defects in dendritic development lead to impaired synapse formation in cultured hippocampal neurons. These neurons were immunostained for synapsin-1 (synaptic vesicle protein) to gauge the number of synapses. Quantification of the colocalized puncta densities was performed along MAP2labeled dendrites. There was a significant decrease in the density of the synapsin-1 puncta in MeCP2-silenced neurons compared to control neurons (Figures 3A,B). Expression of hMeCP2$\mathrm{FL}$ in these $\mathrm{MeCP} 2$-silenced neurons significantly blocked the decrease in density of synapsin-1 puncta (Figure 3B). In contrast, expression of hMeCP2- $\triangle \mathrm{MBD}$ or hMeCP2-T158M in $\mathrm{MeCP} 2$-silenced neurons did not result in any significant changes of the number of synaptic puncta (Figure 3B). These data indicate that an intact MBD domain in MeCP2 is essential for synapse formation in primary hippocampal neurons.

\section{MeCP2 Modulates Spontaneous Calcium Oscillations in Hippocampal Neurons}

To investigate if $\mathrm{MeCP} 2$ is also involved in neurotransmission and synaptic functions, we monitored $\mathrm{Ca}^{2+}$ oscillations in primary hippocampal neurons using a fluorescent calcium dye (X-Rhod-1) to examine the activity of many neurons simultaneously (Figure 4A). Neuronal activity is associated with spontaneous and synchronous rises in intracellular calcium concentration (calcium spikes) in these hippocampal neurons
A

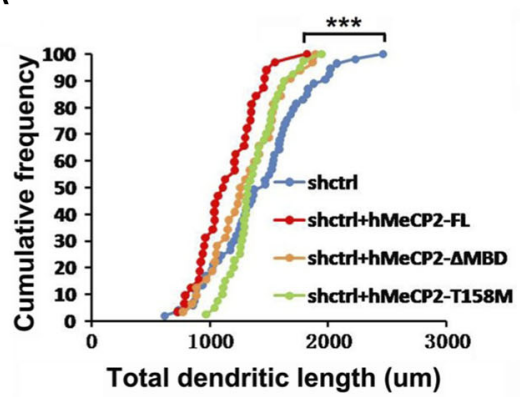

C

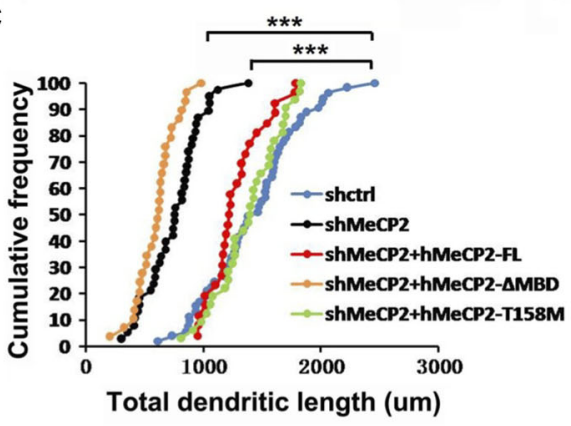

FIGURE 2 | Full length MeCP2 is essential for the maintenance of normal dendritic development in hippocampal primary neurons. $(\mathbf{A}, \mathbf{B})$ Graphs showing total dendritic length $(\mathbf{A})$ and total branch number (B) of cells at 12 DIV after co-infection of various MeCP2 expressing virus (as indicated) in shctrl-expressing cells. (C,D) Graphs showing total dendritic length (C) and total branch number (D) of cells at 12 DIV after co-infection of various MeCP2 expressing virus (as indicated) in
B

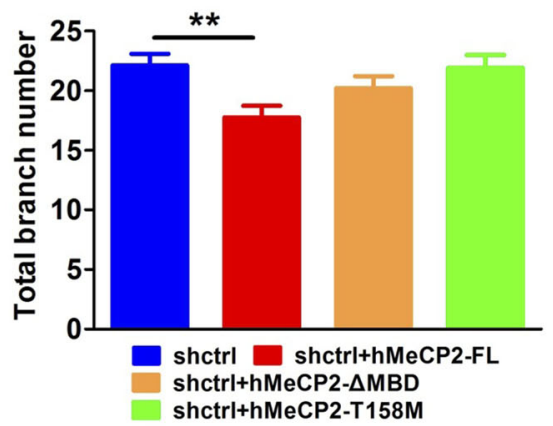

D

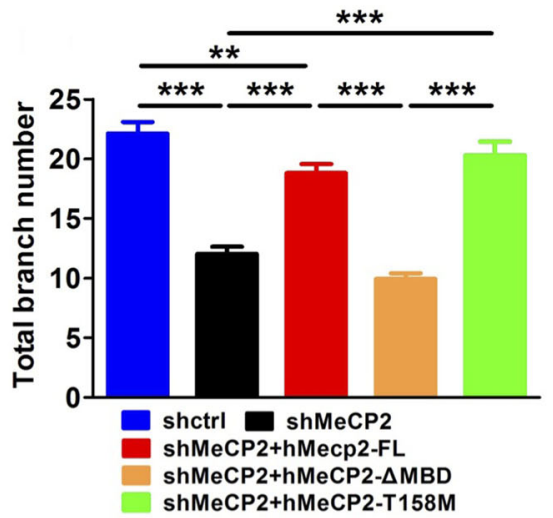

shMeCP2-expressing cells. $\left({ }^{\star *} p<0.01\right.$ and ${ }^{* * *} p<0.001$, one way ANOVA with a post hoc Tukey multiple comparison, number of neurons in (A) and (B): $n=51$ (shctrl), 32 (shctrl + hMeCP2-FL), 33 (shctrl + hMeCP2 - $\triangle \mathrm{MBD}$ ) and 40 (shctrl + hMeCP2-T158M); number of neurons in (C) and (D): $n=51$ (shctrl), 38 (shMeCP2), 26 (shMeCP2 + hMeCP2-FL), 29 (shMeCP2 + hMeCP2 - $\triangle$ MBD), 32 (shMeCP2 + hMeCP2-T158M) from at least 3 batches). 


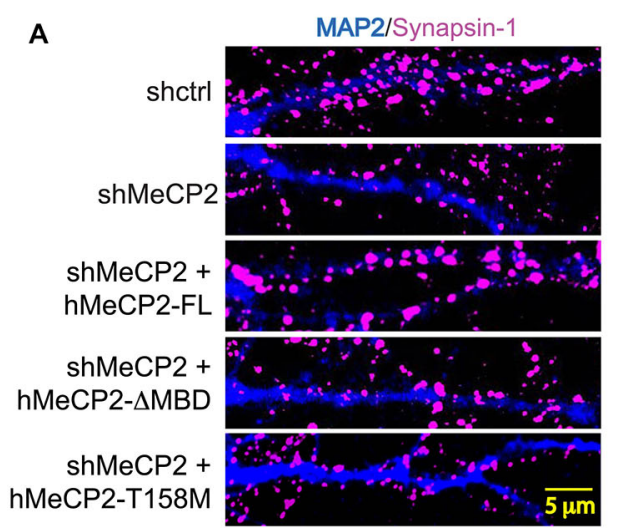

B

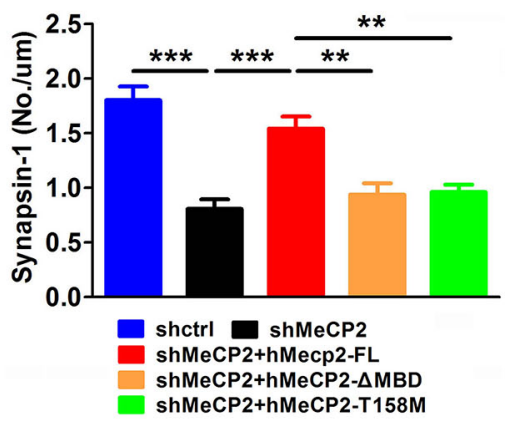

FIGURE 3 | Full length MeCP2 is required for synapse formation. (A) Representative immunostaining images of subcellular distribution of synapsin-1 (pink) in cells infected with shctrl or shMeCP2, co-expression of shMeCP2 with hMeCP2-FL, hMeCP2- $\triangle \mathrm{MBD}$ or hMeCP2-T158M. (B) Quantification of synapsin-1 puncta density per $\mu \mathrm{m}$ dendrite of neurons infected with the constructs as indicated on the graph. ${ }^{* *} p<0.01$ and ${ }^{* \star *} p<0.001$, one-way ANOVA with a post hoc Tukey multiple comparison, number of neurons in (A) and (B): $n=15$ (shctrl), 18 (hMeCP2), 13 (shMeCP2 + hMeCP2-FL), 16 (shMeCP2 + hMeCP2- $\triangle$ MBD), 15 (shMeCP2 + hMeCP2-T158M) from at least 3 batches).
(Leinekugel et al., 1997; Ma et al., 2015). MeCP2-silenced neurons showed increase amplitude but decrease frequency of the spontaneous $\mathrm{Ca}^{2+}$ oscillations compared to control neurons (Figures 4B,C,D,G,H). Cross-correlation analysis demonstrated a higher synchronicity index in $\mathrm{MeCP} 2$ silenced neurons compared to control neurons (Figures $4 \mathrm{E}-\mathbf{H}$ ), indicating stronger synchronization of activity upon MeCP2 silencing.

We then examined the changes in spontaneous $\mathrm{Ca}^{2+}$ oscillations after expression of hMeCP2-FL, hMeCP2- $\triangle \mathrm{MBD}$ or hMeCP2-T158M in MeCP2-silenced neurons. Expression of hMeCP2-FL in MeCP2-silenced neurons rescued MeCP2 deficiency-mediated defects in the amplitude (Figure 4B), the frequency (Figure 4D) and the synchronicity index (Figures 4E,F) of spontaneous $\mathrm{Ca}^{2+}$ oscillations when compared to $\mathrm{MeCP} 2$-silenced neurons alone (Figures $4 \mathrm{~B}-\mathrm{K}$ ). However, expression of neither hMeCP2- $\triangle \mathrm{MBD}$ nor hMeCP2$\mathrm{T} 158 \mathrm{M}$ in $\mathrm{MeCP} 2$-silenced neurons could rescue the abnormal spontaneous $\mathrm{Ca}^{2+}$ oscillation pattern caused by MeCP2 silencing (Figures 4B-K).

\section{Cell Autonomous Effects of MeCP2 in Dendritic Development of Adult Newborn Neurons Requires the MBD Domain}

To examine cell autonomous effects of $\mathrm{MeCP} 2$ in adult newborn neurons, we stereotaxically injected retrovirus with shRNA against a scrambled control sequence or specifically against MeCP2 (Figure 5A) into the dentate gyrus of adult mice. We found significant morphological defects in dendritic arborization in MeCP2-silenced newborn neurons demonstrated by shorter total dendritic length (Figures 5B,C) and fewer branch numbers (Figures 5B,D) compared to control newborn neurons.

To confirm the role of $\mathrm{MBD}$ of $\mathrm{MeCP} 2$ in regulating the dendritic development of newborn neurons in adult brain, we coexpressed hMeCP2-FL or hMeCP2- $\triangle \mathrm{MBD}$ with
shMeCP2 (Figure 6A) in the same newborn granule neurons in vivo. hMeCP2-FL but not hMeCP2- $\triangle \mathrm{MBD}$ expression in $\mathrm{MeCP} 2$-silenced neurons rescued MeCP2 knockdown-mediated dendritic length (Figures 6B,C) and branching (Figures 6B,D) phenotypes. These results confirm the essential role of MBD in dendritic outgrowth of newborn neurons in adult dentate gyrus in vivo.

\section{Discussion}

Disturbances in brain development, neuronal morphology and connectivity were observed in the RTT mouse models (Chen et al., 2001; Belichenko et al., 2008; Lyst and Bird, 2015). Here we provide morphological and functional evidence for the MBD-dependent role of MeCP2 in neuronal development in cultured hippocampal neurons and in newborn granule neurons in adult hippocampus. Our data showed that knockdown of $\mathrm{MeCP} 2$ affects both dendritic length and branch complexity in hippocampal neurons both in vitro (Figure 2) and in vivo (Figure 5). These observations suggest that $\mathrm{MeCP} 2$ is crucial in promoting dendritic growth during early neuronal development. Since morphological alterations in brain architecture are subtle in MeCP2 mutants, there is increasing focus on the functional aspects of synaptic signaling impairments in RTT models. Previous electrophysiological studies on adult mutant MeCP2 mice and MeCP2 knockdown cells revealed an enhanced excitatory neurotransmission (Moretti et al., 2006; Ma et al., 2015) and reduced inhibitory synaptic responses in GABAergic neurons (Chao et al., 2010). Here our results demonstrate a reduction of synapse density in cultured hippocampal neurons upon knocking down MeCP2. Expression of hMeCP2-FL but not the other two MBD mutants in MeCP2-silenced neurons enhanced synapse density, indicating synaptic dysfunction is a critical contributor to RTT phenotypes. Furthermore, it was reported that an imbalance between excitatory and inhibitory 
A
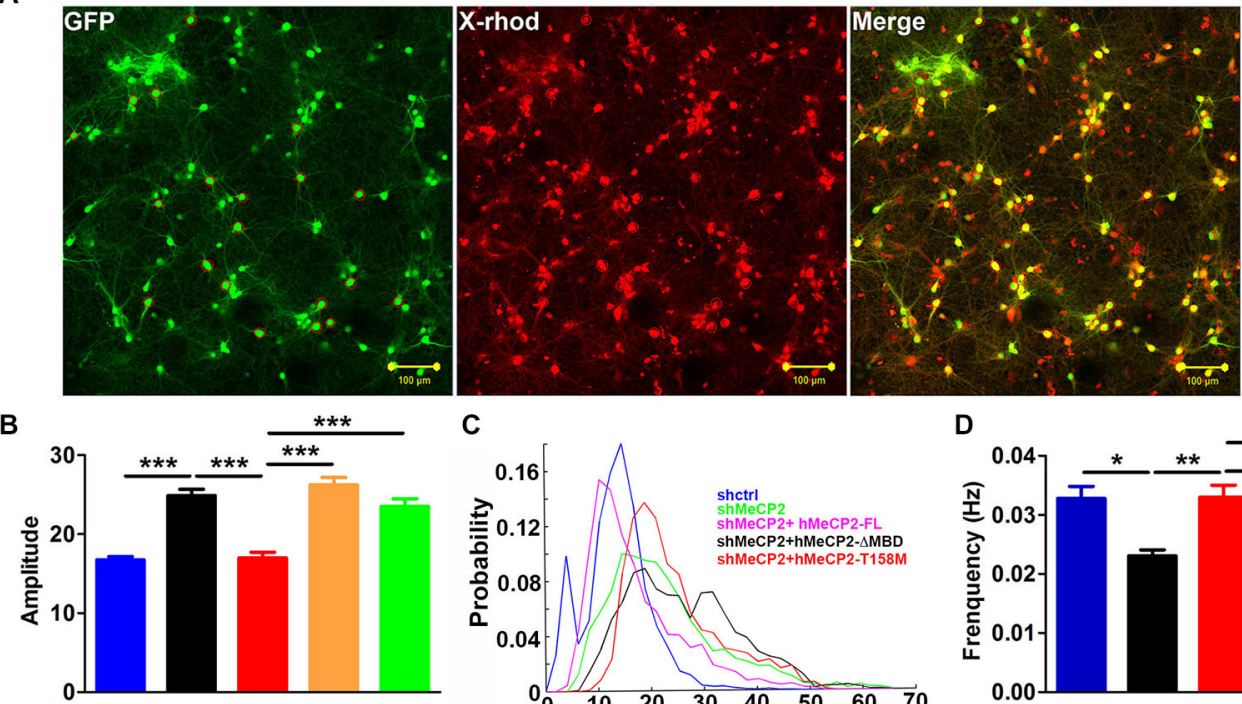

C
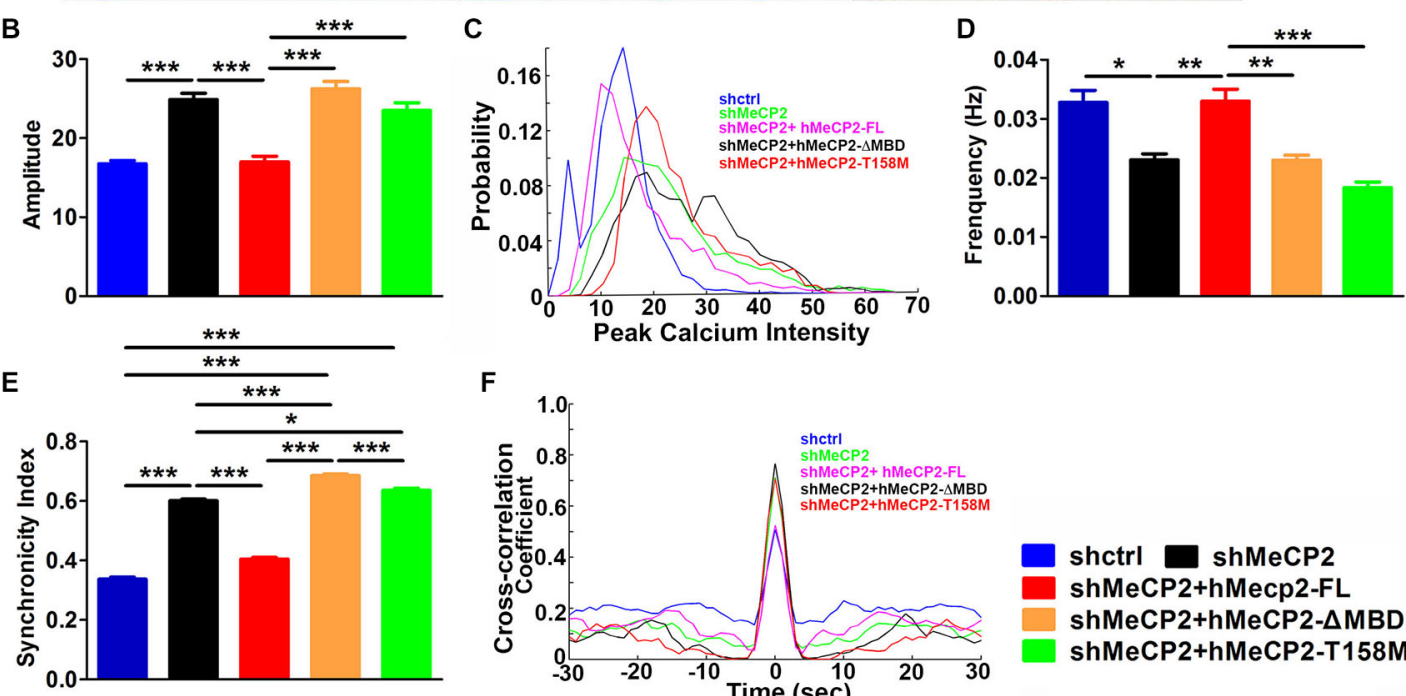

$\mathbf{F}$

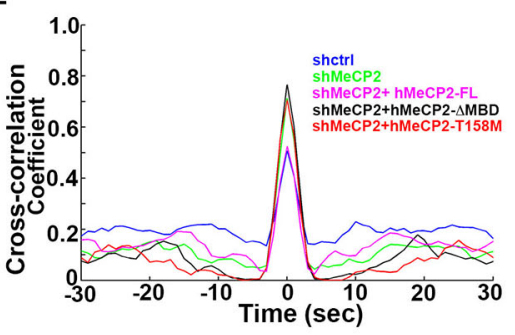

shctrl shMeCP2

shMeCP2+hMecp2-FL

shMeCP2+hMeCP2- $\triangle M B D$

ShMeCP2+hMeCP2-T158M

G

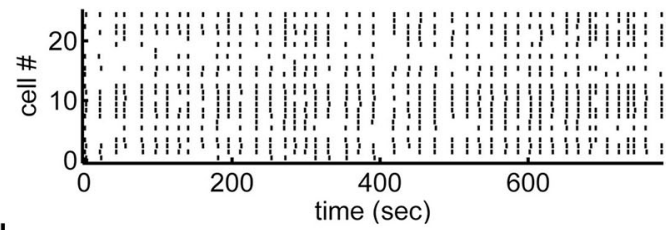

I

shMeCP2+hMeCP2-FL

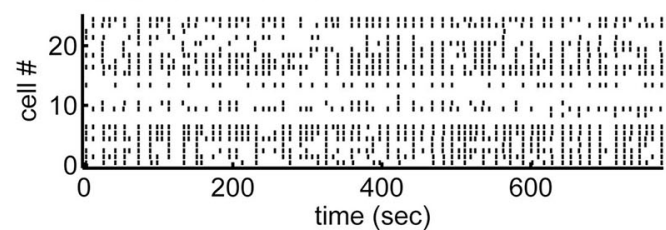

K shMeCP2+hMeCP2-T158M

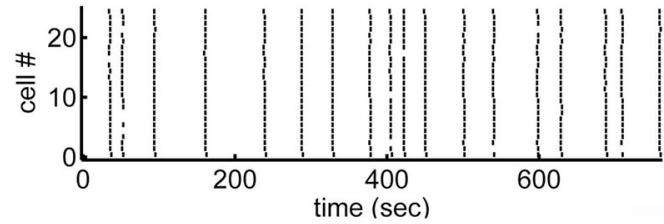

FIGURE 4 | MeCP2 regulates spontaneous calcium oscillations in primary hippocampal neurons. (A) Representative images of neurons pre-infected with different kind of virus (green) and stained with X-Rhod-1 (red) (scale bar = $100 \mu \mathrm{m}$ ). (B-F) Graph shows the amplitude of calcium oscillations (B), the distribution probability of amplitude (C), the frequency (D) and the synchronicity index $(\mathbf{E})$ as well as the cross-correlation coefficient $(\mathbf{F})$ of calcium
H shMeCP2

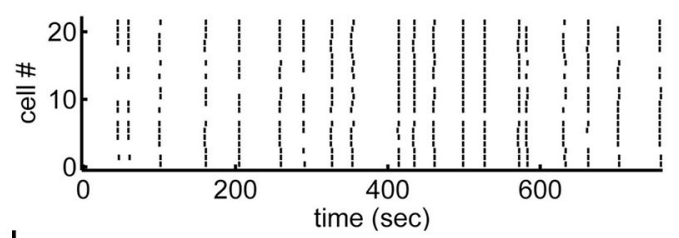

$\mathbf{J}$ shMeCP2+hMeCP2- $\triangle$ MBD

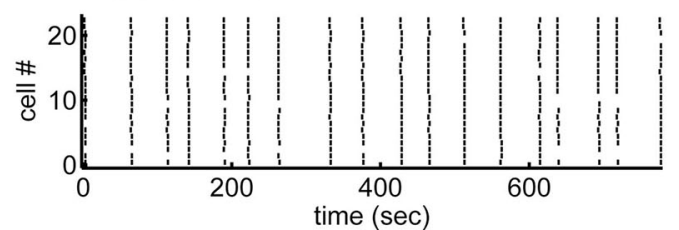

oscillations in neurons infected with the indicated constructs. (G-K) Representative Spike Raster Plot of the different groups of neurons as indicated, showing calcium spikes from $>20$ neurons/plot over a time period of $800 \mathrm{~s}$ as indicated on the $X$-axis. $\left({ }^{\star} p<0.05,{ }^{\star \star} p<0.01\right.$ and ${ }^{\star \star \star} p<0.001$, one-way ANOVA with a post hoc Tukey multiple comparison, $n=120$ neurons from at least 3 batches). 


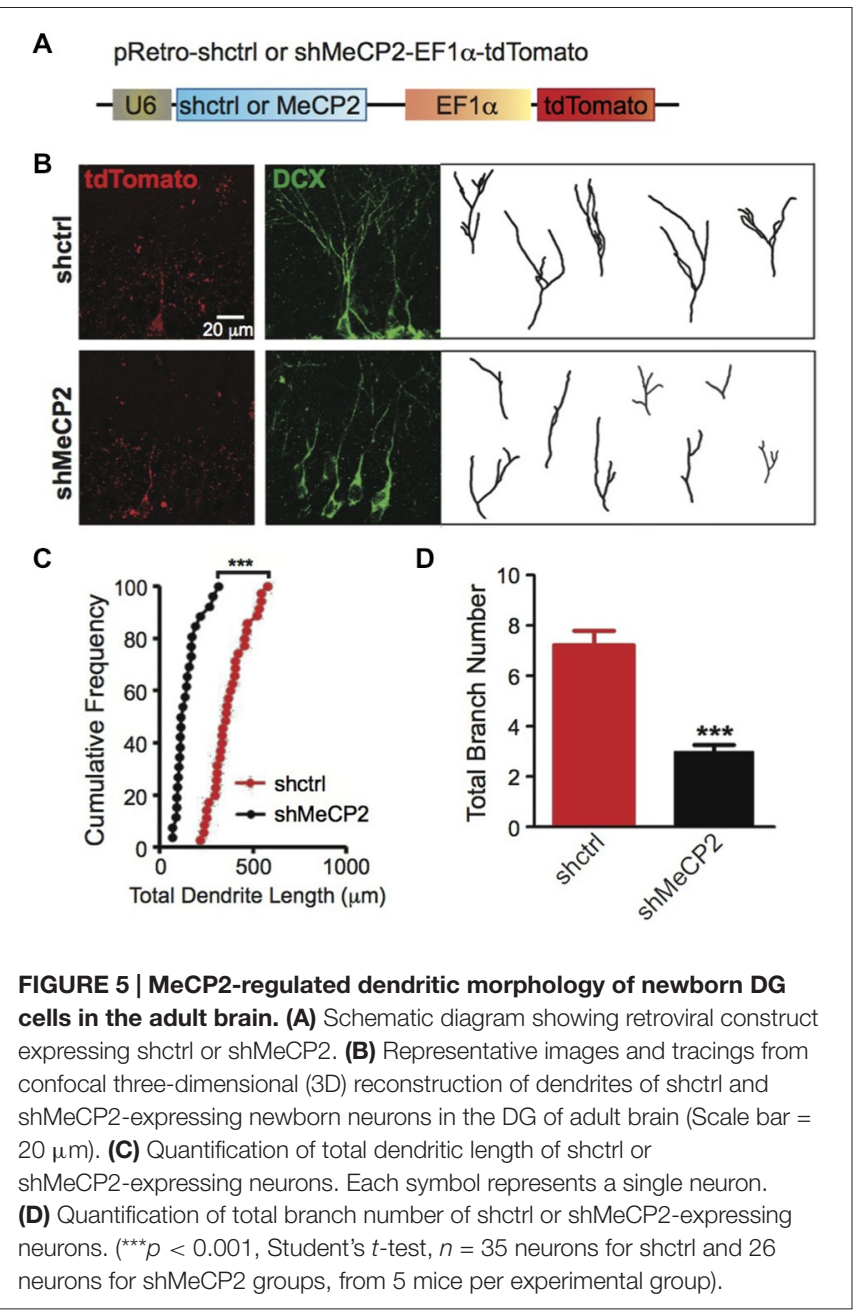

neurotransmission is responsible for several neuropsychiatric phenotypes exhibiting altered learning and memory (Cui et al., 2008) and impaired social behavior (Tabuchi et al., 2007).

Spontaneous $\mathrm{Ca}^{2+}$ oscillations occur as a result of periodical increase and decrease of the free $\mathrm{Ca}^{2+}$ (Garaschuk et al., 2000; Berridge et al., 2003; Okubo et al., 2011), and have been shown to occur in many different neuronal types and at different stages of maturation (Owens et al., 2000; Marchetto et al., 2010; Linde et al., 2011) and are thought to play critical roles in neuronal development and plasticity (Clapham, 1995; Jaskova et al., 2012). A previous study also showed that spontaneous $\mathrm{Ca}^{2+}$ oscillations encode information in their frequency to regulate neurotransmitter expression, channel maturation and neurite extension in spinal neurons ( $\mathrm{Gu}$ and Spitzer, 1995). The pattern of spontaneous $\mathrm{Ca}^{2+}$ oscillations was significantly altered with increased amplitude and decreased frequency in our cultured MeCP2-silenced hippocampal neurons. MeCP2 knockdown also results in synchronized $\mathrm{Ca}^{2+}$ oscillations among neurons, which suggest that the underlying electrical activity, and this functional coupling is correlated to the morphological appearance. However, only hMeCP2-FL but not the other 2 MBD mutants rescued these deficiencies caused by MeCP2 knockdown.
A pRetro-shMeCP2-hMeCP2-FL or $\triangle \mathrm{MBD}$

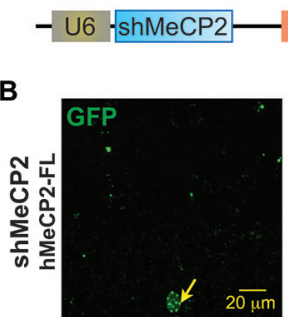
$\mathrm{EF} 1 \alpha$ - EGFP $h M e C P 2-F L / \triangle M B D$ -

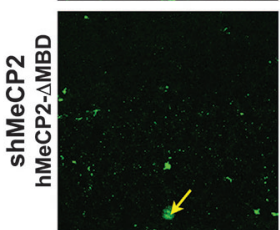

C

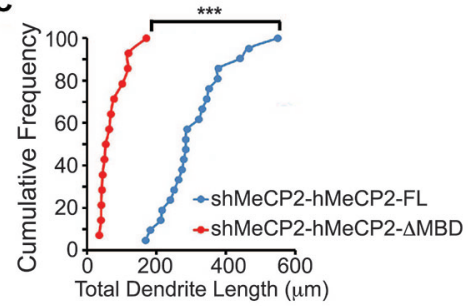

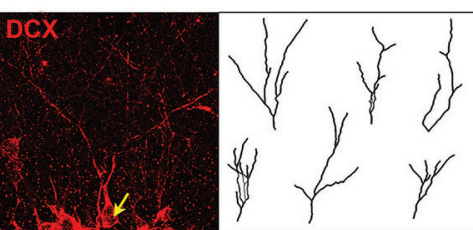
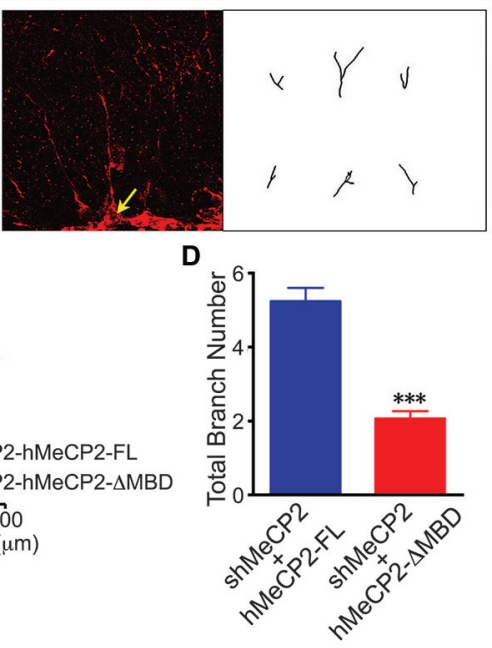

FIGURE 6 | Overexpression of hMeCP2-FL but not hMeCP2- $\triangle$ MBD rescued the defective dendritic morphologies of MeCP2-silenced newborn DG neurons in the adult brain. (A) Schematic diagram showing retroviral construct expressing shMeCP2 together with EGFP-tagged hMeCP2-FL or $\triangle \mathrm{MBD}$. (B) Representative images and tracings from confocal three-dimensional (3D) reconstruction of dendrites of adult newborn neurons expressing either hMeCP2-FL-shMeCP2 or hMeCP2- $\triangle \mathrm{MBD}$-shMeCP2 (Scale bar $=20 \mu \mathrm{m})$. (C) Quantification of total dendritic length of hMeCP2-FL-shMeCP2 or hMeCP2- $\triangle \mathrm{MBD}$-shMeCP2-expressing adult newborn neurons in DG. Each symbol represents a single neuron. (D) Quantification of total branch number of hMeCP2-FL-shMeCP2 or hMeCP2- $\triangle \mathrm{MBD}$-shMeCP2-expressing adult newborn neurons in DG. ${ }^{\left({ }^{* \star} p\right.} p<$ 0.001 Student's $t$-test, $n=21$ neurons for hMeCP2-FL-shMeCP2 and 14 neurons for or hMeCP2- $\triangle \mathrm{MBD}$-shMeCP2 groups, from 5 mice per experimental group).

Patients with mutations in the MBD exhibited more severe clinical features than the other mutations that occurred outside this area (Fabio et al., 2014). Mutation of T158, located at the $\mathrm{C}$ terminus of the MBD in $\mathrm{MeCP} 2$, is one of the most common mutations observed in RTT (Goffin et al., 2011). Approximately $10 \%$ of RTT cases carry the mutation of T158 to methionine or, in rare cases, alanine (Bienvenu and Chelly, 2006). Expression of hMeCP2- $\triangle \mathrm{MBD}$ upon MeCP2 knockdown did not rescue any defects exhibited in $\mathrm{MeCP} 2$ silenced neurons. Intriguingly, expression of hMeCP2-T158M upon MeCP2 knockdown only rescued dendritic arborization (branching), but not the other MeCP2-dysfunction mediated defects that we examined. MeCP2 is named for its ability in binding to specific methylated CpG dinucleotides and $\mathrm{MBD}$ is solely responsible for this binding (Yusufzai and Wolffe, 2000; Ghosh et al., 2010). Furthermore, MBD is highly conserved and appears to be the 
central hub for MeCP2 tertiary structure, forming contacts with the NTD, ID and the TRD. Previous experiments have established the critical role for T158 in the binding of MeCP2 to methylated DNA in vitro (Hansen et al., 2011). Consistently, the T158 mutation was found to have reduced affinity of $\mathrm{MeCP} 2$ for methylated DNA in vivo and $\mathrm{MeCP} 2^{\mathrm{T} 158 \mathrm{~A} / \mathrm{y}}$ mice also presented RTT-like symptoms, including altered anxiety, breathing abnormalities, and impaired learning and memory (Goffin et al., 2011).

Taken together, we found that knocking down MeCP2 in hippocampal neurons resulted in morphological and physiological deficiencies including alterations in dendrite length and branching as well as the number of synaptic puncta and the spontaneous calcium oscillations. These data indicate an essential role of $\mathrm{MeCP} 2$ in synaptic functions. The MeCP2 with T158 mutation only rescued dendritic branching defects while MBD domain truncated deletion of MeCP2 is a complete loss-of function for these MeCP2-dysfunction mediated defects examined. The study of specific mutations or alterations in Mecp2 gene is important for understanding functional implications of these changes in RTT. In addition, our in vivo data also showed cell autonomous effects in dendrites length and branching caused by MeCP2 deficiency that can only be rescued by the full length MeCP2 but not MeCP2- $\triangle \mathrm{MBD}$. Thus, indicating that the MBD domain in

\section{References}

Amir, R. E., Van den Veyver, I. B., Wan, M., Tran, C. Q., Francke, U., and Zoghbi, H. Y. (1999). Rett syndrome is caused by mutations in X-linked MECP2, encoding methyl-CpG-binding protein 2. Nat. Genet. 23, 185-188. doi: 10. $1038 / 13810$

Armstrong, D. D. (2005). Neuropathology of Rett syndrome. J. Child Neurol. 20, 747-753. doi: 10.1177/08830738050200090901

Ballestar, E., Ropero, S., Alaminos, M., Armstrong, J., Setien, F., Agrelo, R., et al. (2005). The impact of MECP2 mutations in the expression patterns of Rett syndrome patients. Hum. Genet. 116, 91-104. doi: 10.1007/s00439-0041200-0

Belichenko, N. P., Belichenko, P. V., Li, H. H., Mobley, W. C., and Francke, U. (2008). Comparative study of brain morphology in Mecp2 mutant mouse models of Rett syndrome. J. Comp. Neurol. 508, 184-195. doi: 10.1002/cne. 21673

Berridge, M. J., Bootman, M. D., and Roderick, H. L. (2003). Calcium signalling: dynamics, homeostasis and remodelling. Nat. Rev. Mol. Cell Biol. 4, 517-529. doi: $10.1038 / \mathrm{nrm} 1155$

Bienvenu, T., and Chelly, J. (2006). Molecular genetics of Rett syndrome: when DNA methylation goes unrecognized. Nat. Rev. Genet. 7, 415-426. doi: 10. 1038/nrg1878

Chao, H.-T., Chen, H., Samaco, R. C., Xue, M., Chahrour, M., Yoo, J., et al. (2010). Dysfunction in GABA signalling mediates autism-like stereotypies and Rett syndrome phenotypes. Nature 468, 263-269. doi: 10.1038/nature09582

Chapleau, C. A., Calfa, G. D., Lane, M. C., Albertson, A. J., Larimore, J. L., Kudo, S., et al. (2009). Dendritic spine pathologies in hippocampal pyramidal neurons from Rett syndrome brain and after expression of Rett-associated MECP2 mutations. Neurobiol. Dis. 35, 219-233. doi: 10.1016/j.nbd.2009. 05.001

Chen, R. Z., Akbarian, S., Tudor, M., and Jaenisch, R. (2001). Deficiency of methylCpG binding protein-2 in CNS neurons results in a Rett-like phenotype in mice. Nat. Genet. 27, 327-331. doi: 10.1038/85906

Clapham, D. E. (1995). Calcium signaling. Cell 80, 259-268. doi: 10.1016/00928674(95)90408-5
MeCP2 is critical for normal dendrite development in vitro and in vivo.

\section{Author Contributions}

$\mathrm{NZ}$ performed most in vitro and in vivo experiments, analyzed data and wrote the manuscript; DM designed all experiments, performed some in vitro and in vivo experiments and calcium imaging experiments; WYL designed and made all MeCP2 WT and mutant constructs; JH and AVD analyzed data for calcium imaging; TC provided inputs to the project; ELG initiated and directed the entire study, designed experiments, analyzed data and wrote the manuscript.

\section{Funding and Disclosure}

This work was supported by Competitive Research Program (CRP) funds from National Research Foundation, Singapore, GlaxoSmithKline (GSK) Academic Center of Excellence (ACE) Award and Abbott Nutrition to ELKG.

\section{Acknowledgments}

We thank members of the Goh lab for sharing of reagents and expertise.

Cui, Y., Costa, R. M., Murphy, G. G., Elgersma, Y., Zhu, Y., Gutmann, D. H., et al. (2008). Neurofibromin regulation of ERK signaling modulates GABA release and learning. Cell 135, 549-560. doi: 10.1016/j.cell.2008.09.060

Fabio, R. A., Colombo, B., Russo, S., Cogliati, F., Masciadri, M., Foglia, S., et al. (2014). Recent insights into genotype-phenotype relationships in patients with Rett syndrome using a fine grain scale. Res. Dev. Disabil. 35, 2976-2986. doi: 10. 1016/j.ridd.2014.07.031

Garaschuk, O., Linn, J., Eilers, J., and Konnerth, A. (2000). Large-scale oscillatory calcium waves in the immature cortex. Nat. Neurosci. 3, 452-459. doi: 10. $1038 / 74823$

Ghosh, R. P., Nikitina, T., Horowitz-Scherer, R. A., Gierasch, L. M., Uversky, V. N., Hite, K., et al. (2010). Unique physical properties and interactions of the domains of methylated DNA binding protein 2. Biochemistry 49, 4395-4410. doi: 10.1021/bi9019753

Goffin, D., Allen, M., Zhang, L., Amorim, M., Wang, I.-T. J., Reyes, A.-R. S., et al. (2011). Rett syndrome mutation MeCP2 T158A disrupts DNA binding, protein stability and ERP responses. Nat. Neurosci. 15, 274-283. doi: 10.1038/nn. 2997

Gu, X., and Spitzer, N. C. (1995). Distinct aspects of neuronal differentiation encoded by frequency of spontaneous Ca2+ transients. Nature 375, 784-787. doi: $10.1038 / 375784 \mathrm{a} 0$

Hansen, J. C., Wexler, B. B., Rogers, D. J., Hite, K. C., Panchenko, T., Ajith, S., et al. (2011). DNA binding restricts the intrinsic conformational flexibility of MeCP2. J. Biol. Chem. 286, 18938-18948. doi: 10.1074/jbc.m111. 234609

Heckman, L. D., Chahrour, M. H., and Zoghbi, H. Y. (2014). Rett-causing mutations reveal two domains critical for MeCP2 function and for toxicity in MECP2 duplication syndrome mice. Elife 3:e02676. doi: 10.7554/eLife. 02676

Jaskova, K., Pavlovicova, M., and Jurkovicova, D. (2012). Calcium transporters and their role in the development of neuronal disease and neuronal damage. Gen. Physiol. Biophys. 31, 375-382. doi: 10.4149/gpb_2012_053

Leinekugel, X., Medina, I., Khalilov, I., Ben-Ari, Y., and Khazipov, R. (1997). Ca ${ }^{2+}$ oscillations mediated by the synergistic excitatory actions of GABA(A) and NMDA receptors in the neonatal hippocampus. Neuron 18, 243-255. doi: 10. 1016/s0896-6273(00)80265-2 
Linde, C. I., Baryshnikov, S. G., Mazzocco-Spezzia, A., and Golovina, V. A. (2011). Dysregulation of $\mathrm{Ca}^{2+}$ signaling in astrocytes from mice lacking amyloid precursor protein. Am. J. Physiol. Cell Physiol. 300, C1502-C1512. doi: 10. 1152/ajpcell.00379.2010

Lyst, M. J., and Bird, A. (2015). Rett syndrome: a complex disorder with simple roots. Nat. Rev. Genet. doi: 10.1038/nrg3897. [Epub ahead of print].

Ma, D., Yoon, S.-I., Yang, C.-H., Marcy, G., Zhao, N., Leong, W.-Y., et al. (2015). Rescue of Methyl-CpG binding protein 2 Dysfunction-induced defects in newborn neurons by Pentobarbital. Neurotherapeutics doi: 10.1007/s13311015-0343-0. [Epub ahead of print].

Marchetto, M. C. N., Carromeu, C., Acab, A., Yu, D., Yeo, G. W., Mu, Y., et al. (2010). A model for neural development and treatment of rett syndrome using human induced pluripotent stem cells. Cell 143, 527-539. doi: 10.1016/j.cell. 2010.10.016

Marshak, S., Meynard, M. M., De Vries, Y. A., Kidane, A. H., and Cohen-Cory, S. (2012). Cell-autonomous alterations in dendritic arbor morphology and connectivity induced by overexpression of MeCP2 in Xenopus central neurons in vivo. PLoS One 7:e33153. doi: 10.1371/journal.pone.0033153

Moretti, P., Levenson, J. M., Battaglia, F., Atkinson, R., Teague, R., Antalffy, B., et al. (2006). Learning and memory and synaptic plasticity are impaired in a mouse model of Rett syndrome. J Neurosci 26, 319-327. doi: 10. 1523/JNEUROSCI.2623-05.2006

Neul, J. L., Kaufmann, W. E., Glaze, D. G., Christodoulou, J., Clarke, A. J., Bahi-Buisson, N., et al. (2010). Rett syndrome: revised diagnostic criteria and nomenclature. Ann. Neurol. 68, 944-950. doi: 10.1002/ana.22124

Ng, T., Ryu, J. R., Sohn, J. H., Tan, T., Song, H., Ming, G.-L., et al. (2013). Class 3 semaphorin mediates dendrite growth in adult newborn neurons through Cdk5/FAK pathway. PLoS One 8:e65572. doi: 10.1371/journal.pone.0065572

Nguyen, M. V. C., Du, F., Felice, C. A., Shan, X., Nigam, A., Mandel, G., et al. (2012). MeCP2 is critical for maintaining mature neuronal networks and global brain anatomy during late stages of postnatal brain development and in the mature adult brain. J. Neurosci. 32, 10021-10034. doi: 10.1523/JNEUROSCI. 1316-12.2012

Okubo, Y., Kanemaru, K., and Iino, M. (2011). Imaging of $\mathrm{Ca}^{2+}$ and related signaling molecules and investigation of their functions in the brain. Antioxid. Redox Signal. 14, 1303-1314. doi: 10.1089/ars.2010.3367
Owens, D. F., Flint, A. C., Dammerman, R. S., and Kriegstein, A. R. (2000). Calcium dynamics of neocortical ventricular zone cells. Dev. Neurosci. 22, 25-33. doi: 10.1159/000017424

Ricceri, L., De Filippis, B., and Laviola, G. (2013). Rett syndrome treatment in mouse models: searching for effective targets and strategies. Neuropharmacology 68, 106-115. doi: 10.1016/j.neuropharm.2012.08.010

Ricciardi, S., Boggio, E. M., Grosso, S., Lonetti, G., Forlani, G., Stefanelli, G., et al. (2011). Reduced AKT/mTOR signaling and protein synthesis dysregulation in a Rett syndrome animal model. Hum. Mol. Genet. 20, 1182-1196. doi: 10. 1093/hmg/ddq563

Shahbazian, M. D., and Zoghbi, H. Y. (2002). Rett syndrome and MeCP2: linking epigenetics and neuronal function. Am. J. Hum. Genet. 71, 1259-1272. doi: 10. $1086 / 345360$

Stancheva, I., Collins, A. L., Van den Veyver, I. B., Zoghbi, H., and Meehan, R. R. (2003). A mutant form of MeCP2 protein associated with human Rett syndrome cannot be displaced from methylated DNA by notch in Xenopus embryos. Mol. Cell 12, 425-435. doi: 10.1016/s1097-2765(03)00276-4

Tabuchi, K., Blundell, J., Etherton, M. R., Hammer, R. E., Liu, X., Powell, C. M., et al. (2007). A neuroligin-3 mutation implicated in autism increases inhibitory synaptic transmission in mice. Science 318, 71-76. doi: 10.1126/science.11 46221

Yusufzai, T. M., and Wolffe, A. P. (2000). Functional consequences of Rett syndrome mutations on human MeCP2. Nucleic Acids Res 28, 4172-4179. doi: $10.1126 /$ science. 1146221

Conflict of Interest Statement: The authors declare that the research was conducted in the absence of any commercial or financial relationships that could be construed as a potential conflict of interest.

Copyright (c) 2015 Zhao, Ma, Leong, Han, VanDongen, Chen and Goh. This is an open-access article distributed under the terms of the Creative Commons Attribution License (CC BY). The use, distribution and reproduction in other forums is permitted, provided the original author(s) or licensor are credited and that the original publication in this journal is cited, in accordance with accepted academic practice. No use, distribution or reproduction is permitted which does not comply with these terms. 